\title{
Entrelacs
}

Cinéma et audiovisuel

\section{Chris Cunningham : quand le vidéoclip questionne l'imaginaire générique adolescent}

\section{Aleksandra Borowiec}

\section{(2) OpenEdition}

\section{Journals}

Édition électronique

URL : http://journals.openedition.org/entrelacs/341

DOI : 10.4000/entrelacs.341

ISSN : 2261-5482

Éditeur

Éditions Téraèdre

Édition imprimée

Pagination : 11-22

ISSN : 1266-7188

Référence électronique

Aleksandra Borowiec, «Chris Cunningham : quand le vidéoclip questionne l'imaginaire générique adolescent », Entrelacs [En ligne], 9 | 2012, mis en ligne le 22 octobre 2012, consulté le 20 avril 2019.

URL : http://journals.openedition.org/entrelacs/341 ; DOI : 10.4000/entrelacs.341

Ce document a été généré automatiquement le 20 avril 2019.

Tous droits réservés 


\title{
Chris Cunningham : quand le vidéoclip questionne l'imaginaire générique adolescent
}

\author{
Aleksandra Borowiec
}

1 Chris Cunningham, un artiste visuel britannique, commence son aventure avec le clip musical en 1996 avec Second Bad Vibel. Réalisé pour le groupe de musique électronique Autechre, il met en scène les convulsions violentes d'un robot quadrupède. Dans ce premier essai, tout en apprenant les techniques du métier, Cunningham définit déjà son style et ses centres d'intérêt. En concepteur de créatures pour des films comme Alien ${ }^{3}$ (D. Fincher, 1992), Judge Dredd (D. Cannon, 1995), ou Artificial Intelligence: A.I. (S. Spielberg, 2001), il divulgue sa fascination pour l'anatomie en y appliquant avec soin un rendu robotique hyperréaliste.

2 L'esthétique de Second Bad Vibel rappelle une vidéo de test clinique, brouillée par les interférences cathodiques, ce qui provoque une sensation de malaise et de danger chez le spectateur. Nous ne savons pas, en effet, si les problèmes avec l'image viennent du dispositif de diffusion ou de la machine en question qui risque de s'échapper du laboratoire et nous attaquer. Le travail de la texture de la vidéo et l'utilisation des images parasites deviendra aussi une marque de fabrique de Cunningham qui poursuit son autoformation en tant que réalisateur en acceptant toutes les propositions. Mais c'est grâce à la collaboration avec Aphex Twin ${ }^{1}$, le maestro de la musique électronique expérimentale, que Cunningham infiltre officiellement la principale plateforme de diffusion de clips qu'est MTV avec son univers grouillant de créatures menaçantes.

\section{De la féminité domptée à l'altérité monstrueuse}

3 La chaine musicale MTV (Music Television) voit le jour le $1^{\text {er }}$ août 1981. Le refrain du premier vidéoclip diffusé, « Video Killed the Radio Star » du groupe The Buggles, explicite la mission de ses concepteurs. Dorénavant, c'est l'image accompagnant le morceau qui devient le véhicule de la popularité de l'artiste musical. Comme l'explique Lisa A. Lewis : 
"L'industrie musicale cherchait désespérément un nouveau mode de promotion économique et efficace pour faire ressortir leur produit du lot. [...] Ni la radio, ni les concerts, ne pouvait fournir une exposition nationale simultanée possible via MTV. [...] La chaine câblée s'est avérée l'outil de vente le plus puissant jamais possédé ${ }^{2}$ " par les maisons de disque.

4 Le public cible de MTV étant les jeunes de 12 à 34 ans $^{3}$ sexuellement éveillés, l'érotisme et l'attractivité physique commencent à devenir des éléments clés de l'imagerie diffusée via les clips musicaux. En conséquence, l'industrie musicale voit naître les stars dont le sexappeal et la photogénie prévalent sur le talent musical. Nous pouvons citer ici l'exemple de Madonna qui, consciente de ses limites en tant que chanteuse et danseuse, saisit tout de suite l'enjeu de promotion visuelle via le clip. D'ailleurs, le début de la célébrité de l'interprète concorde avec le lancement de MTV.

5 L'audience adolescente nourrit alors sa perception de la sexualité avec des figures érotiques proposées via MTV. Madonna, le précurseur de tendances musicales et visuelles dans le monde de la pop, incarne à tour de rôle des fantasmes masculins, de la mariée prête à sa nuit de noces («Like a Virgin », 1984) jusqu’à la dominatrix dans le monde de vices collectifs ("Erotica », 1992) ou une chanteuse de bar à prostituées ("Secret », 1994). Madonna introduit les comportements sexuels bannis dans la société bien pensante en mettant à l'image les attitudes homéotiques féminines, le sexe de groupe, les travestis, le sadomasochisme... Toutefois, moyennant l'esthétique de magazines de mode signée JeanBaptiste Mondino ou Fabien Baron ${ }^{4}$, Madonna réussit à aseptiser et dompter la chair au service de la représentation publicitaire. Cette tendance est retenue aujourd'hui par des interprètes comme Lady Gaga, qui puisent volontiers dans l'esthétique de la presse à scandale («Paparazzi », 2009) ou du genre filmique sexploitation (Telephone, 2010) pour construire leur identité visuelle.

D’une manière générale, du rock au rap, les clips musicaux se caractérisent par la présence régulière d'une multitude de jolies filles, membres volontaires et sexuellement accessibles d'une congrégation festive rassemblée autour des interprètes. La beauté des corps stimule le public adolescent en établissant ainsi les archétypes d'une sexualité moderne à l'altérité modérée et communément acceptable.

7 Dans cet univers de corporéité disciplinée, les clips de Cunningham soulèvent plusieurs peurs et tabous concernant la chair et reflétant les angoisses adolescentes via la figure du monstre - le «freak». En découvrant les différences génitales entre les sexes, l'importance réelle ou imaginaire de leur taille, les codes esthétiques liés à la pilosité, les jeunes ont du mal à définir la norme sociale acceptable de la sexualité. Aussi, il y a une notion de l'étrangéité, donc de monstruosité, dans la perception de l'autre sexe et ses attributs génitaux. En suivant l'explication de Leslie Fiedler, « un jeune homme regardant une vulve peut appréhender son propriétaire en tant que monstre par défaut, tandis que une jeune femme regardant son pénis peut le trouver monstre par excès. Ou, en y réfléchissant, il peut se sentir monstre par excès, elle, monstre par défaut. ${ }^{5}$ " De ces différences naît peut-être une mythologie de l'hermaphrodisme - le monstre double qui effraie par l'impossibilité d'association à aucun genres ni rôles sociaux et qui véhicule avec lui le questionnement sur les penchants homoérotiques.

8 Le tabou de l'homosexualité et du transsexualisme est très présent dans la culture rap et hip-hop. L'image de la masculinité confirmée conjugue la richesse matérielle voyante (bijoux en or et diamants, voitures de luxe, villas, champagne, etc.) et la performance 
sexuelle prouvée par la présence de plusieurs femmes en petite tenue. Paradoxalement, l'idéal féminin exposé dans les clips rap s'approche de la figure du travesti dans l'exagération des formes corporelles (les seins, la bouche et les fesses), l'utilisation abondante du maquillage et des accessoires (faux ongles, talons aiguilles) ainsi que le recours visible à la chirurgie esthétique. Cunningham se sert de cette contradiction pour concevoir Windowlicker ${ }^{6}$, un pastiche de l'imagerie machiste des clips rap.

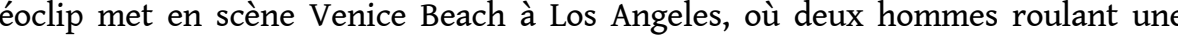
Mazda décapotable "tunée ${ }^{7}$ » cherchent des femmes pour subvenir à leurs besoins sexuels. Ils réussissent à aborder deux filles en petite tenue au bord de la route mais se font refuser violemment ${ }^{8}$. En pleine dispute, leur voiture est percutée par un überphallus : une limousine blanche de plus de quarante mètres de long. En sort un homme au visage d'Aphex Twin arborant un rictus inquiétant immobile. Le nouveau venu séduit deux femmes en dansant d'une façon explicite. Les visages de deux femmes morphent à l'instar de celui de l'homme et elles le rejoignent, au milieu d'autres femmes au même visage masculin dans la limousine. En suivent des scènes de dance où les deux jeunes hommes découvrent une femme au beau corps dont la tête s'avère être particulièrement repoussante. Le clip finit sur une séquence au ralenti dans laquelle Aphex Twin secoue et fait exploser une bouteille de champagne. Le liquide abondant asperge les danseuses en bikini blanc. Les lents travellings rapprochés permettent de voir en détail le mouvement de la graisse des fesses et des seins féminins.

Les pin-ups de Windowlicker effraient par leur ambiguïté sexuelle. Leur corps étant immédiatement associable aux clubs de strip-tease, leur visage est celui de Richard D. James, un homme barbu d'une attractivité physique moyenne. En effet, Cunningham fait porter à tous les personnages le même masque en silicone et latex qui intègre à la perfection le reste du corps en produisant un effet d'illusion complet, obtenu avec la possibilité de clignotement des paupières.

11 En ôtant à l'objet, sa dimension de simulacre théâtral (qui distingue bien le visage du masque), le réalisateur rend la peur viscérale, sans laisser au spectateur la possibilité de repli dans l'artifice. Dans la lignée d'utilisation des effets spéciaux au cinéma qui substituent au lieu de suggérer, les femmes dans Windowlicker ne se déguisent pas dans le but de représenter, mais demeurent les êtres monstrueux à tête démoniaque et renvoient ainsi aux peurs primaires, vers l'origine religieuse du masque. M. Bernard explique que « l'étymologie même du mot est d'ailleurs fort révélatrice : celui-ci vient en effet du mot latin de basse époque (643 après J.-C.) masca qui désigne la sorcellerie (...). [Le terme dénote] la présence, ou, mieux, l'incarnation d'une puissance démonique apparente ${ }^{9} »$. C'est alors une force malicieuse qui semble s'incarner dans ces sorcières au corps de rêve qui mènent à la tentation la sexualité hétéro de l'homme en mettant en question l'utilisation de l'artifice dans l'amplification de l'attractivité sexuelle de la femme. Car si les opérations esthétiques peuvent souligner les attributs féminins, elles peuvent également cacher une identité étrangère monstrueuse qui menace la virilité.

La figure du monstre et de la possession nous renvoie également vers un des archétypes du cinéma d'horreur qu'est le corps étranger, figurant en anglais sous le nom d'alien et utilisé depuis des décennies dans des milliers de films d'épouvante. Mais à l'instar des figures mythiques comme le Terminator (J. Cameron, 1984) ou l'Alien (R. Scott, 1979), Chris Cunningham décline à la sauce cyberpunk l'idéologie rousseauienne, qui oppose l'organique, le naturel et le mécanique. Le réalisateur puise dans l'imagerie de l'horreur viscérale dans «Sheena Is A Parasite » (The Horrors, 2006) où l'actrice Samantha Morton, 
connue pour son rôle dans le drame cyberpunk Minority Report (S. Spielberg, 2002), est secouée frénétiquement au rythme stroboscopique. Lors du refrain qui dénonce que "Sheena est un parasite », la femme lève sa jupe pour faire jaillir vers la caméra ce qui semble être des intestins ou des tentacules. Comme la créature du film de Ridley Scott qui nait du thorax humain, la femelle monstrueuse de Cunningham bouscule la frontière entre l'intestinal et le reproductif, menace l'ordre social et biologique établi en effaçant l'homme de l'équation. C'est l'hybride qui effraie par annonce d'une ère post-humaine dépourvue de repères génériques, mettant en question les comportements sexuels dans le contexte de reproduction incertaine et le sort violent de la progéniture.

\section{La violence de grandir}

13 Le réalisateur britannique se sert de l'imagerie horrifique de l'enfant dans le premier clip qu'il réalise pour Aphex Twin : "Come To Daddy ». Dans un décor urbain populaire de la cité Thamesmead à l'est de Londres, les enfants au visage souriant de Richard D. James attaquent une femme âgée qui promène son chien et un homme dans le parking souterrain. En surgissant des zones d'ombre et cassant tout sur leur passage, six petites créatures semblent répondre à l'appel lancé d'un poste télé au milieu des poubelles qui émet l'image d'un visage distordu. Au moment où la femme âgée s'approche du poste, celui-ci « accouche » d'un monstre-père imposant et effroyable qui rassemble autour de lui une descendance tout aussi épouvantable.

Dans l'interview qui figure dans le livret accompagnant son DVD ${ }^{10}$, Chris Cunningham raconte l'origine du concept de "Come To Daddy » : "Je me souvenais d'être poursuivi dans les bois par un enfant de neuf ans, alors que j'en avais environ seize. J'étais sur ma bécane et lui avait un marteau. C'était humiliant et je le détestais. A l'âge de seize ans tu ne veux pas finir pulvérisé par un garçon de neuf ans, au cas où les filles regardent $!^{11}$ ». Dans l'entretien pour le magazine Dazed and Confused, le réalisateur britannique se rappelle: «J'ai eu une enfance la plus idyllique et libérale possible. Peut-être du fait que je n'avais aucune restriction, je pouvais dessiner des scènes de bandes dessinées étranges, sexuelles, et maman disait quelque chose comme "C'est très joli mon chéri." Tu ne pouvais pas l'énerver. [...] J'étais très paranoïaque. Mes premiers souvenirs de l'école primaire était d'être assis là en pensant "l'instit pense que je suis un branleur", donc j'essayais très fort de bien travailler. Je supposais que les gens pensaient que je ne valais rien. ${ }^{12}$ »

Les personnages incarnent alors peut-être le mal-être physique, les paranoïas enfantines, mais aussi, de par leur monstruosité, la gêne par rapport à son corps, si caractéristique de la période de puberté. A travers ses gamins diaboliques, Cunningham semble questionner le statut sociosexuel de l'être mineur. Comme l'explique Leslie Fiedler, «il y a [...] le problème de la sexualité enfantine créée par la nature bisexuelle, polymorphique et perverse des enfants pré-pubères, aggravé par la position changeante sur leur viabilité sexuelle du monde adulte. (...) Pourtant, quels que soient les codes de sa culture, préfreudiens ou post-freudiens, répressifs ou permissifs, l'enfant sent une anomalie monstrueuse entre sa nature érotique et les attentes de la société. $»^{13}$

16 Contrairement aux figures cinématographiques des enfants maléfiques ${ }^{14}$ où l'horreur réside dans le choc entre l'apparence extérieure et la réalité des actes, les petits du clip d'Aphex Twin constituent une image totale de l'anomalie. Aussi, à la différence de «Sheena Is A Parasite» mettant en scène la maternité monstrueuse, les enfants de 
« Come To Daddy » apparaissent en tant que fruit de la paternité cathodique, arborant les caractéristiques du genre masculin. En effet, au lieu de faire appel aux vrais enfants, Cunnigham utilise les personnes adultes de petite taille. Associant alors une construction anatomique robuste et une pilosité bien visible au faciès diabolique et barbu d'Aphex Twin, le réalisateur conteste la perception de l'enfance en tant que période innocente et asexuelle.

La question de l'ignorance de la sexualité adolescente par les parents et la répugnance ressentie vis-à-vis de son corps en pleine mutation se manifeste aussi dans un autre clip réalisé par Cunningham pour Aphex Twin : «Rubber Johnny ». La signification du titre à double sens (le nom d'un personnage, mais aussi «une capote» en argot anglais) est littéralement illustrée lors de la séquence-titre, où nous pouvons voir l'image inversée d'un préservatif enlevé du pénis en érection. Tourné entièrement en vision nocturne, le vidéoclip met en scène Johnny, un jeune homme en fauteuil roulant atteint probablement d'hydrocéphalie, qui passe ses journées enfermé dans la cave par ses parents qui descendent occasionnellement pour gronder leur fils à cause du bruit. Son seul compagnon, un chihuahua, est témoin de la dance de Johnny sur son fauteuil roulant. Tout seul dans la chambre vide, le jeune homme déformé abuse de la drogue, produit des flashes de lumière avec ses mains et écrase son visage sur le verre ce qui dénature encore plus sa physionomie. Mais dès que le père invisible et autoritaire ouvre la porte, il voit son fils immobile sur le fauteuil en train de balbutier des mots incompréhensibles à son chien.

Laissés à eux-mêmes, les jeunes laissent libre cours à la violence, la destruction et les gestes obscènes, refusent le physique angélique et aseptisé qu'y attribue la société adulte et expriment la monstruosité sexuelle malgré les tentatives de répression de la part de l'autorité. Car il y a quelque chose d'effrayant dans ce corps adolescent qui se réveille, dont les parties poussent, durcissent pour se rétrécir ensuite. En conséquence, il devient plus compréhensible le fait que Chris Cunningham traite l'être humain comme un amas de parties anatomiques transformables et associables, de pièces détachées en pleine mutation. Là où il y a la honte de cette chair disproportionnée qui n'obéit pas, Cunningham propose une libération par la technologie. L'homme est le point de départ, un être imparfait par sa corporéité. Le vidéaste essaie alors d'approcher l'existence humaine et sa sexualité incontrôlable vers la perfection d'existence des objets techniques.

\section{Les machines du plaisir et le plaisir des machines}

Cunnigham explore les enjeux du désir vécu en dehors de la réalité charnelle dans « All Is Full of Love », le vidéoclip réalisé pour Björk qui consolide la réputation du réalisateur ${ }^{15}$. Le clip raconte l'histoire d'une rencontre sexuelle de deux robots gynomorphiques dans un espace confiné qui rappelle un laboratoire immaculé de "Bad Second Vibel ». Encore une fois, dans l'univers blanc et stérile, les machines s'expriment, mais cette fois, au lieu de secousses menaçantes des carcasses, si caractéristiques des clips du réalisateur britannique, le spectateur assiste aux préparatifs posés du coït mécanique. Les deux automates identiques au visage de la chanteuse islandaise, dépourvus de parties génitales, procèdent à des caresses et des baisers prudents.

Toutefois, « All Is Full of Love » se met en opposition à l'imagerie des machines sexuelles, le fantasme aussi bien masculin que féminin. Effectivement, les hommes rêvent d'une partenaire infatigable, prête à satisfaire le désir sexuel à n'importe quel moment et 
dépourvue de ses propres besoins dans le domaine. Ces chimères bioniques constituent d'ailleurs l'objet de plusieurs films comme The Pleasure Machines (R.V.Garcia, P. Hunt, 1967) ou Cherry 2000 (S. De Jarnatt, 1987), dans lesquels l'homme peut posséder une machine d'une façon sexuellement gratifiante. Les femmes portent un intérêt érotique sur les machines qui représentent un potentiel sexuel égalitaire, libéré de toute connotation reproductive. A défaut des hommes attentionnés à l'orgasme de leurs partenaires, les femmes cherchent leurs sex-machines, les étalons électriques qui peuvent leur faire l'amour indéfiniment. Nous retrouvons ce type de songes dans les films pornographiques des fucking machines ${ }^{16}$, des instruments mécaniques dotés de plusieurs stimulateurs simultanés. Les appareils mis à disposition des participantes comprennent aussi bien les dispositifs de la stimulation clitoridienne comme sybian, la tronçonneuse à langues en latex ou le vibromasseur vulvaire, que les différents types de godemichés mécaniques transférant la force rotationnelle (les perceuses ou les moteurs). Ils existent aussi des combinaisons variées utilisant des jets d'eau ou des caméras internes. Le coït se déroule souvent sous le contrôle de la femme qui reste en possession de différentes télécommandes. Ainsi, elle administre le dosage du plaisir et règle le fonctionnement de la machine sur ses besoins jusqu'au climax.

21 Cette fois, Cunningham questionne le discours cyberpunk des chantres du bionisme qui cherchent à augmenter la performance sexuelle à l'aide de prothèses mécaniques et va au-delà de la conception moderne du corps transformé. Car quand la performance est là, où en est-on au niveau de l'intimité ? Par quel biais passe la satisfaction érotique ? Dans "All Is Full of Love", le réalisateur interroge la justesse de l'affirmation du titre, se permet une réflexion portée sur la place de l'affection dans la société contemporaine obsédée par la compétitivité. L'intimité de l'acte, assurée en apparence par l'enfermement de l'atelier, est impossible. En effet, la rencontre est rendue possible et assistée par des kilomètres de câbles et d'autres machines qui alimentent les deux robots, soudent leurs connectiques, les lubrifient... Aussi, en assistant à la rencontre sensuelle entre Björk et son double, le spectateur est en réalité témoin d'une séance d'autostimulation et non d'un accouplement de deux organismes. Le vidéoclip traite donc de la solitude d'un être sexuellement fragilisé, hanté par la performativité, le sexe mécanique dépourvu d'émotion.

David Le Breton suggère que « si le corps est un symbole de la société [...], toute menace sur sa forme affecte symboliquement le lien social. Les limites du corps dessinent à leur échelle l'ordre moral et signifiant du monde. Penser le corps est une autre manière de penser le monde et le lien social : un trouble introduit dans la configuration du corps est un trouble introduit dans la cohérence du monde. ${ }^{17}$ » Ainsi, en s'inspirant de différentes figures de l'altérité corporelle, les vidéoclips de Chris Cunningham constituent une représentation relativement complète de la confusion générique et des tourments sexuels adolescents : la peur de l'autre sexe, l'incompréhension de son propre corps, le manque d'identité et l'impossibilité de communiquer avec le monde.

23 Alors, à l'image du chaos intérieur qui ronge le jeune public de MTV, les créations de Chris Cunningham saturent les quelques minutes de diffusion sur la chaine avec l'image stroboscopique ${ }^{18}$, mêlant le réel et l'étrange, le concret et l'abstrait. La rapidité du récit visuel rend presque impossible le déchiffrement complet des images figuratives et cadres parasites. De même, le spectateur n'est pas sûr de voir, il en a l'impression. Dans l'univers cunninghamien rien n'est certain ni défini. 
Aussi, si les œuvres de Cunningham trouvent un tel écho auprès de la génération MTV habitué des clips musicaux, c'est parce que le vidéaste recours aux caractéristiques narratives de deux genres cinématographiques proches du public adolescent : le cinéma d'horreur et la pornographie. Le réalisateur revisite les motifs connus en renvoyant au spectateur la mise en image et en mouvement des peurs et des préjugés liés à l'Autre. Comme l'explique Carol Clover, « le fait que les conventions de l'horreur sont parodiés si facilement et si souvent voudrait suggérer que, malgré des variations individuelles, ses structures de base d'aperception restent fixes et fondamentales... L'art du film d'horreur, comme l'art de la pornographie, est principalement l'art de l'interprétation et reste compris comme tel par le public compétent. Un exemple particulier peut posséder des traits originaux, mais sa qualité en tant que film d'horreur réside dans la façon dont il livre le cliché. $»^{19}$

Le realisateur britannique teste les possibilités formelles du vidéoclip tout en respectant ses codes (image esthétisante, musique omniprésente, rythme accéléré du montage) ce qui lui permet de transmettre sa vision monstrueuse du monde au milieu d'autres clips diffusés en un bloc hétéroclite.

\section{NOTES}

1. Aphex Twin étant le pseudonyme artistique de Richard D. James, un artiste musical irlandais. Dans l'article, nous utiliseront les deux noms d'une manière synonymique.

2. LEWIS A. Lisa, "Gender Politics and MTV. Voicing the Difference », éd. Temple University Press, Philadelphia, 1990, p. 23-24, traduit par nos soins

3. Op. cit., p. 19.

4. Mondino et Baron sont deux photographes de mode mondialement connus. Jean Baptiste Mondino, collaborateur régulier de Jean-Paul Gauthier, réalise en 1991 «Justify My Love », un clip en noir et blanc mettant en scène des expériences érotiques collectives dans un hôtel. La vidéo est la première œuvre audiovisuelle à être bannie de MTV. Fabien Baron, fort de l'expérience dans les magazines comme GQ, Vogue Italia ou Interview, rejoint Madonna en 1992 sur le plateau d' « Erotica ». Le vidéoclip retrace les sessions photo pour le livre de la chanteuse intitulé « Sex ». « Erotica » est le deuxième clip dans l'histoire à être interdit par MTV.

5. FIEDLER Leslie, «Freaks. Myths and Images of the Secret Self», éd. Simon and Schuster, New York, 1978, p. 32, traduit par nos soins.

6. Windowlicker désignant à la fois « lèche-vitrine », une personne qui, faute de moyens financiers, ne fait que convoiter la marchandise exposée, mais aussi « retardé » en argot anglais (d'après la définition d'Urban Dictionary: http://www.urbandictionary.com/define.php? term=windowlicker). Cunningham s'inspire du double sens du titre du morceau musical pour mettre en scène l'incapacité de deux jeunes hommes à courtiser les femmes.

7. Tuning, une modification de la voiture afin d'augmenter sa performance ou son apparence, fait partie entre autres de la culture hip-hop et s'inscrit dans l'esthétique flamboyante d'accessoirisation de l'individu.

8. La version officielle du clip dure $10 \mathrm{~min} 45 \mathrm{sec}$ (générique de fin comprise). Pendant le premières $3 \min 50 \mathrm{sec}$. de la «séquence de drague », 127 gros mots sont utilisés, ce qui fait une 
moyenne d'une insulte par deux secondes. Pour pouvoir être diffusé sur MTV, la version «bipée » est préparée. Toutefois, MTV Networks Europe se voit condamnée à une amende de 255 mille livres pour la diffusion de la version non-censurée avant $21 \mathrm{~h}$ («MTV fined $£ 255 \mathrm{k}$ for offensive material », de Mark Sweney, paru dans Guardian le 04/06/2008).

9. In Dictionnaire encyclopédique du théâtre, sous la direction de Michel Corvin, éd. Bordas, Paris, 1991, p. 539.

10. Directors Label Volume 2: The Work of Director Chris Cunningham, éd. PalmDVD, New York, 2003.

11. Traduit par nos soins.

12. «In Full Effect», de Callum McGeoch, paru dans Dazed and Confused en avril 1999, traduit par nos soins.

13. FIEDLER Leslie, Freaks. Myths and Images of the Secret Self, éd. Simon and Schuster, New York, 1978, p. 30, traduit par nos soins.

14. Nous pouvons citer ici l'exemple des personnages enfantins de Village of the Damned (W. Rilla, 1960), de Damien de The Omen (R. Donner, 1976), mais aussi les enfants de Das weisse Band (M.Haneke, 2009).

15. «All Is Full of Love » a gagné le prix du meilleur clip expérimental et des meilleurs effets spéciaux lors de MTV Video Music Awards et a été nominé aux Grammy Awards dans la catégorie du meilleur clip musical. En 2008, les spectateurs de MTV2 l'ont élu le meilleur vidéoclip de tous les temps. Il se trouve également dans la collection permanente du Museum of Modern Art de New York.

16. Plusieurs sites pornographiques offrent la possibilité de visionner des films, la principale plateforme restant http://www.fuckingmachines.com. Néanmoins l'article «Les sex-machines sont déjà là " de Javier Cid, paru dans Courrier International n 941 en novembre 2008 et décrivant Fuckzilla, la sex-machine ultime, constitue également une source d'information intéressante.

17. LE BRETON David, La chair à vif, collection Traversées sous la direction de Pascal Dibie, éd. A.M. Métailié, Paris, 1993, p. 316.

18. Montées toujours en concordance avec le rythme du morceau qu'elles accompagnent, plusieurs créations de Cunningham attaquent le spectateur avec un nombre impressionnant d'images par seconde (la moyenne d'environ deux images par seconde pour « Come To Daddy ", idem pour «Bad Second Vibel»). La violence de l'image accélérée est utilisée également dans la bande annonce de son DVD, ainsi que dans le menu principal de sa publication.

19. SMITH W. Andy, "'These children that you spit on': horror and generic hybridity ", In Monstrous Adaptations: Generic and Thematic Mutations in Horror Film, éd. Manchester University Press, Manchester \& New York, 2007, p. 84-85, traduit par nos soins.

\section{AUTEUR}

\section{ALEKSANDRA BOROWIEC}

Doctorante 